
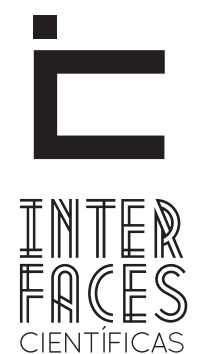

EDUCAÇÃo

ISSN IMPRESSO 2316-333X

E-ISSN 2316-3828

DOI-10.17564/2316-3828.2016v5n1p95-106

\title{
UMA UTOPIA IRREALIZÁVEL? O DEBATE SOBRE A EDUCAÇÃO INCLUSIVA NA ALEMANHA
}

Romana Castro Zambrano ${ }^{1}$

\section{RESUMO}

Em 2008, com a ratificação da Convenção sobre os Direitos das Pessoas com Deficiência da Organização das Nações Unidas (ONU), a Alemanha se comprometeu a estabelecer um sistema educacional inclusivo. Essa decisão política causou um debate polêmico na mídia alemã que continua até hoje. Nesse debate, notam-se diversas conceitualizações da educação inclusiva que, por um lado, refletem a opinião da sociedade e, por outro, influenciam a percepção social da educação inclusiva. 0 presente estudo propõe uma análise des- sas conceitualizações com base na Análise Crítica do Discurso (ACD) e na Linguística Cognitiva (LC). Com isso, visa-se a apontar para algumas problemáticas socioculturais na realização da educação inclusiva.

\section{PALAVRAS-CHAVE}

Educação inclusiva. Alemanha. Análise Crítica do Discurso. Linguística Cognitiva. 


\section{ABSTRACT}

In 2008, by the ratification of the UN Convention on the Rights of Persons with Disabilities, Germany has undertaken to establish an inclusive education system. This political decision has caused a controversial discussion in the German media which continues to this day. In this debate, various conceptualizations of inclusive education can be noticed. These conceptualizations, on the one hand, reflect the society's views, and, on the other hand, influence the social perception of inclusive education. This study proposes an analysis of these conceptualizations based

\section{RESUMEN}

En 2008, con la ratificación de la Convención sobre los Derechos de las Personas con Discapacidad de las Naciones Unidas, la Alemania se comprometió a establecer un sistema de educación inclusivo. Esta decisión política causó un debate polémico en los medios alemanes que se mantiene hasta los días actuales. En este debate, se observaron diferentes conceptualizaciones de la educación inclusiva. Estas conceptualizaciones, por un lado, reflejan las opiniones de la sociedad y, por el otro lado, influyen en la percepción social sobre la inclusión. Este artículo propone on Critical Discourse Analysis (CDA) and Cognitive Linguistics (CL). The purpose of this is to point out some socio-cultural issues concerning the realization of inclusive education.

\section{KEYWORDS}

Inclusive education. Germany. Critical Discourse Analysis. Cognitive Linguistics. un análisis de estas conceptualizaciones con base en el Análisis Crítico del Discurso (ACD) y la Lingüística Cognitiva (LC). De este modo, se pretende señalar algunas cuestiones socioculturales en cuanto a la realización de la educación inclusiva.

\section{PALABRAS CLAVE}

Educación inclusiva. Alemania. Análisis Crítico del Discurso. Lingüística Cognitiva. 


\section{INTRODUÇ̄̃̃O}

A inclusão, como um ideal ético-filosófico, representa a ideia de uma sociedade heterogênea na qual a singularidade de cada pessoa é reconhecida e aceita (KRÖG, 2005). Levando esse conceito a um nível mais concreto, no âmbito escolar, entende-se como inclusão assegurar a igualdade de direitos com respeito a uma educação qualitativa e inclusiva para todos. Destarte, não somente a exclusão, mas também a segregação escolar de pessoas com necessidades educacionais especiais, contrariam o ideal da inclusão.

Desde a última década do século passado, documentos internacionais, como a Declaração Mundial sobre Educação para Todos (1990) e, sobretudo, a Declaração de Salamanca (1994), promoveram a divulgação e o estabelecimento do conceito da educação inclusiva em nível mundial. Em muitos países europeus, iniciou-se uma mudança nos sistemas escolares para garantir a inclusão das pessoas com necessidades educacionais especiais. Não obstante, naquela época, não todos os países do continente europeu participaram dessa mudança (SCHUMANN, 2007). Assim, a Alemanha adotou o conceito da educação inclusiva somente com a ratificação da Convenção sobre os Direitos das Pessoas com Deficiência da Organização das Nações Unidas (ONU) (BRASIL, 2012) em 2008. Com essa ratificação, o país se comprometeu a reconhecer

[...] o direito das pessoas com deficiência à educação. Para efetivar esse direito sem discriminação e com base na igualdade de oportunidades, os Estados Partes assegurarão sistema educacional inclusivo em todos os níveis, bem como o aprendizado ao longo de toda a vida [...]. (BRASIL, 2012, p. 48).

Porém, na atualidade, ainda constatam-se dificuldades na realização dessas exigências. Um dos obstáculos é o fato de que a responsabilidade administrativa e legislativa não cabe ao Estado federal, mas aos Estados federados. Portanto, as leis escolares re- gionais apresentam grandes diferenças no avanço da aplicação e também na interpretação da Convenção (MISSLING; ÜCKERT, 2014).

Além disso, é o próprio sistema escolar alemão que estorva a realização da educação inclusiva, pois se trata de um sistema segregativo, orientado pela filosofia de que todos os alunos devem receber uma educação correspondente às suas capacidades individuais. Dessa maneira, a partir do nível secundário, existem diferentes tipos de escolas: 0 Gymnasium, a Realschule e a Hauptschule. Consequentemente, após o ensino primário de quatro anos, as crianças são avaliadas e, em concordância com seu desempenho, as escolas primárias elaboram um parecer, contendo uma recomendação para um desses tipos de escolas: os alunos com as melhores notas devem frequentar um Gymnasium e os com as piores notas uma Hauptschule. Entre si, os tipos de escolas contam com bastantes diferenças quanto às disciplinas ensinadas e também quanto ao diploma de conclusão.

Destaca-se que somente o Gymnasium, com uma duração de oito a nove anos, oferece a secundária II que qualifica diretamente para o ensino superior em uma universidade ${ }^{2}$. Tanto a Realschule como a Hauptschule, com uma duração média de seis anos, qualificam unicamente para estudar em uma escola profissionalizante ou, dependendo das notas, para frequentar a secundária II em ainda outros tipos de escolas (VIOTTI, 2014). A Gesamtschule (escola comum) oferece uma alternativa a esse sistema, posto que ela une os três tipos de escolas, oferecendo os três referentes diplomas de conclusão. No contexto da inclusão, desenvolveu-se uma variante da Gesamtschule: a Gemeinschaftsschule (escola comunitária), que se orienta pela ideia da escola inclusiva. Porém, a maioria dessas escolas não inclui a secundária II (RATZKI, 2011).

2. Na Alemanha, a educação secundária se divide em dois ciclos: a secundária I e a secundária II. Estes ciclos correspondem aos níveis II e III da Classificação Internacional Normalizada da Educação (UNESCO, 2012). 
Em virtude disso, pode-se constatar que as oportunidades no mercado laboral variam em dependência do tipo de escola frequentado. Fora dessas escolas regulares, a Alemanha dispõe de um sistema de escolas especiais, chamadas de escolas de apoio. Consoante o Estado federado, essas escolas oferecem diferentes focos de apoio. Por exemplo, em Berlim, existem escolas com os focos ouvir, ver, idioma, aprender, desenvolvimento mental, desenvolvimento físico e motor, desenvolvimento emocional e social, deficiência autista e uma escola para alunas e alunos doentes (BERLIM, 2004). Em 2012, 6,6\% dos alunos foram classificados como alunos com necessidades educacionais especiais e $3,3 \%$ dos alunos frequentam escolas de apoio desde a escola primária (AUTORENGRUPPE..., 2014). Considerando essas características do sistema de educação alemão com seu alto nível de segregação, pode-se constatar que ele representa uma ideia diametralmente oposta à ideia do ensino inclusivo. Portanto, não surpreende que a decisão política a favor da educação inclusiva iniciou uma discussão no discurso midiático. Esse debate se intensificou em 2011, quando a Conferência dos Ministros da Cultura emitiu um documento norteador para realizar a inclusão escolar (ALEMANHA, 2011).

Em vista disso, o estudo presente visa a retratar a significação do conceito da inclusão no discurso midiático alemão, focando efeitos sociais quanto à reorganização do sistema educativo. Para constituir o corpus da análise, foram coletados artigos de jornais alemães nas suas versões on-line com relevância nacional de 2011 a 2015. Em particular, consideraram-se os jornais BILD.de (BILD), ZEIT ONLINE (DIE ZEIT), SZ.de (Süddeutsche Zeitung) e FAZ.NET (Frankfurter Allgemeine Zeitung). Orientando-se teórica e metodologicamente pela Análise Crítica do Discurso (ACD) e pela Linguística Cognitiva (LC), a pesquisa se centra na questão da conceitualização da educação inclusiva no discurso midiático.

\section{A ANÁLISE CRÍTICA DO DISCURSO E A LINGUÍSTICA COGNITIVA}

Nos anos 1980, desenvolveu-se uma vertente da análise do discurso interdisciplinar, baseado na linguística: a Análise Crítica do Discurso (ACD). A ACD parte do pressuposto de que o discurso, manifestado em textos escritos, orais ou em qualquer outra forma de comunicação, mantém uma relação dialética com a sociedade. Isto é, que a sociedade está influenciada pelo discurso e vice-versa (FAIRCLOUGH; WODAK, 1997). Para explicar essa inter-relação, van Dijk (2003) argumenta que é imprescindível considerar uma instância intermediária entre o discurso e a sociedade: a cognição social.

Sob o termo cognição social entende-se um sistema mental compartilhado pelos membros de certas comunidades e grupos. Esse sistema compõe-se por vários subsistemas, tais como atitudes, ideologias, normas ou valores. Um subsistema ao qual se atribui um status especial é o saber, visto que este é reconhecido pela sociedade como a verdade e, portanto, constitui a sua realidade social (VAN DIJK 2002). Considerando que os grupos dominantes em uma sociedade têm um maior acesso aos meios de comunicação de massa, esses grupos possuem mais facilidade de determinar e construir verdades sociais que grupos minoritários. Correspondentemente, o discurso sempre está marcado por certas relações de poder.

Em face disso, a ACD visa a revelar ideologias naturalizadas, desigualdades sociais, injustiça e estruturas de dominância e poder no discurso, alertando para a situação das oprimidas e dos oprimidos. Esse engajamento político-emancipatório se alinha à filosofia de Jürgen Habermas, adotando um posicionamento crítico quanto ao uso da linguagem como instrumento de dominação (WODAK, 1995). Em consequência, a ACD persegue com seus estudos também um fim pedagógico, o fim de criar uma consciência crítica do discurso, apontando para a 
importância de questionar e refletir o discurso e o uso da linguagem (FAIRCLOUGH, 2006).

Nesse sentido, o aspecto crítico da ACD justifica também seu enfoque interdisciplinar, visto que o discurso indica um envolvimento da linguagem com o político, o social e o histórico. Wodak (1995) salienta que a recorrência a outras áreas de conhecimento não deve acontecer de modo arbitrário, mas quando resulta necessária para a análise de um determinado objeto de pesquisa.

Para esses fins, na introdução deste estudo, situou-se o objeto da pesquisa em seu contexto histórico-social, contemplando as políticas públicas e o sistema escolar da Alemanha. Além disso, lembrando da interface sociocognitiva, opta-se na análise pela aplicação de uma teoria linguística que, pela fusão com as ciências cognitivas, representa uma área transdisciplinar: a Linguística Cognitiva (LC).

Segundo Hart (2013), a LC aplicada à ACD mostra como construções linguísticas refletem a ideologia e como a (re)produzem, pois a LC não somente foca o lado dos emitentes, mas, também, dos receptores. Em consonância com as bases teóricas da LC, a relação entre a linguagem e a realidade não é direta, mas mediada pela cognição. Destarte o significado é "visto como uma construção cognitiva através da qual o mundo é apreendido e experienciado" (FERRARI, 2014, p. 14). A linguagem, então, é considerada a parte visível dos processos mentais da construção do significado.

Uma das primeiras teorias que influenciaram significativamente o desenvolvimento da LC foi a teoria das metáforas cotidianas (LAKOFF; JOHNSON, 1980), distanciando-se da noção tradicional da metáfora como adorno retórico pela afirmação de que as línguas em si são metafóricas. Nesse pensamento, a mente humana organiza conhecimentos de uma maneira metafórica, explicando conceitos abstratos por meio de conceitos mais concretos. Enquanto inicial- mente a motivação dessas explicações foi entendida principalmente por experiências físicas (LAKOFF; JOHNSON, 1999), nos últimos anos a LC passou a enfatizar também o aspecto sociocultural em suas teorias (FRANK ET AL., 2008).

Neste estudo, focaliza-se a teoria cognitiva da integração (ou mesclagem) conceitual (FAUCONIIER; TURNER, 2002). Conforme Ferrari (2014, p. 120), essa operação mental "pode ser considerada a origem da nossa aptidão para novos sentidos”. A integração conceitual descreve processos mentais nas quais se junta e mistura informação de dois (ou mais) espaços mentais por meio de uma projeção parcial. Em consequência, gera-se um novo espaço mental com estrutura emergente. Destarte, a teoria não somente explica os processos mentais subjacentes a metáforas, mas também a analogias, metonímias, categorizações e outros. (FAUCONNIER; TURNER, 2008).

Isso posto, a teoria da integração conceitual analisa, por exemplo, a expressão vírus de computador, destacando que um conceito da área de saúde é projetado a um conceito da informática. Também, construções sintáticas são investigadas a partir dessa teoria, assim, sublinha-se na frase Paris é o coração da França a combinação de um conceito geográfico e um conceito da anatomia, gerando, dessa maneira, o novo sentido de que Paris tem uma função vital para a França (FERRARI, 2014). Visto que o objeto de pesquisa deste estudo é o discurso e que este abrange uma dimensão superordenada ao texto, à frase ou à unidade lexical, a teoria da integração conceitual é aplicada com o fim de identificar uma rede de conceitos semelhantes que determinam o conceito da educação inclusiva ao nível discursivo.

\section{A CONCEITUALIZAÇ̃̃O DA EDUCAÇÃO INCLUSIVA COMO UM PROJETO IRREAL}

Como resultado principal da análise, pode-se constatar que a mídia, em muitos casos, contesta o 
fator realizável da educação inclusiva. Essa conceitualização discursiva efetiva-se por meio da integração conceitual, combinando a educação inclusiva com vários conceitos diferentes, cujos aspectos semânticos comuns podem ser determinados como o irreal e o imaginário. Em seguida, mostrar-se-ão alguns exemplos encontrados no corpus. Em vista de que o significado se desenvolve no seu contexto discursivo, os exemplos serão explicados a partir de fragmentos discursivos.

Um conceito, que faz parte da rede de significações, é a educação inclusiva como utopia. Segundo o dicionário Duden online (DUDENREDAKTION 2016), a palavra utopia, em alemão Utopie, refere-se, em um sentido geral, a um "plano que parece irrealizável" ou uma "ideia sem fundamento real”. Essa conceitualização se apresenta no seguinte recorte com referência à inclusão social total e sua precursora: a escola inclusiva.

(1) Correspondentemente, constatam-se reações alérgicas quando uma "escola para todos" mal planejada é apresentada como a precursora de uma inclusão social total, uma utopia social, na qual - como devemos nos imaginar (lema da inclusão aqui: “Vamos ver!”) todos têm os mesmos direitos para participar em todos os lugares. (FAZ.NET, 10/06/2014; tradução própria).

(Original em alemão: Entsprechend allergisch fallen die Reaktionen aus, wenn eine schlampig begründete "Schule für alle" auch noch als Vorreitereiner totalen gesellschaftlichen Inklusion angepriesen wird, einer sozialen Utopie, bei der - wie immer man sich das vorzustellen hat (Inklusionsmotto hier: „Schauen wir mal!") - alle überall gleichberechtigt mit von der Partie sein werden.)

Nota-se que o caráter irreal da inclusão social total como utopia social é ainda reforçado pelo verbo imaginar. Lembrando que a Alemanha se comprometeu com a ratificação da Convenção da ONU para um sistema educacional inclusivo (BRASIL, 2012), convém frisar que esse trecho classifica as exigências desse documento como utópico. Concomitantemente, nesse fragmento do discurso a utopia não se refere à inclusão em geral, mas à inclusão social total, uma especificação que encontramos de modo semelhante no recorte seguinte:

(2) Melhorias concretas são possíveis, mas é quase inevitável que a inclusão total continuará a ser uma utopia. Até essa abordagem concreta modesta deve se preocupar com problemas de aplicação. Porque nem todos nós colocamos sempre em prática, o que achamos teoricamente correto [...] (ZEIT ONLINE, 15/07/2015; tradução própria).

(Original em alemão: Konkrete Verbesserungen sind möglich, doch eine vollständige Inklusion wird fast zwangsläufig eine Utopie bleiben. Sogar dieser bescheidene, konkrete Ansatz muss Durchsetzungsprobleme befürchten. Denn nicht immer tun wir alle das ganz praktisch, was wir theoretisch richtig finden [].)

Além de conceitualizar a inclusão total como utopia, destaca-se nesse fragmento a avaliação positiva dela como teoria, enquanto a viabilidade é questionada. Desse modo, ressalta-se o aspecto irrealizável da utopia. Com uma afirmação parecida, também deparamos no próximo trecho:

(3) Claro, a ideia da inclusão é maravilhosa. Quem não deseja que as crianças com e sem deficiência aprendam de uma maneira natural juntas e que todos recebam um apoio individual? Infelizmente, de um ideal elevado não resulta uma realidade ideal.

E, como a história tem mostrado, sobretudo as belas utopias têm, às vezes, feios efeitos colaterais. (ZEIT ONLINE, 27/03/2014; tradução própria).

(Original em alemão: Natürlich ist die Idee der Inklusion wunderbar. Wer wünschte sich nicht, dass Kinder mit und ohne Handicap ganz selbstverständlich miteinander lernen und alle individuell gefördert werden? Leider folgt aus einem hehren Ideal noch keine ideale Wirklichkeit.

Und wie die Geschichte lehrt, haben gerade schöne Utopien mitunter hässliche Nebenwirkungen.)

Embora sendo inicialmente avaliada de uma maneira positiva, por causa da palavra ideia, essa avaliação se restringe à teoria da inclusão. Destaca-se que a palavra maravilhosa (wunderbar em alemão), considerando seu substantivo maravilha (Wunder em ale- 
mão), também está relacionada a um acontecimento que não faz parte da realidade cotidiana. Outrossim, a palavra deseja associa-se com algo positivo (ainda) inalcançado que, portanto, também não corresponde à realidade. 0 contraste entre um ideal elevado e a realidade ideal alinha-se no seguinte esquema de conceitualização: contrastando uma teoria com sua realização na prática. Enfim, o artigo declara a inclusão de uma bela utopia, alertando para seus feios efeitos colaterais. Com isso, a inclusão na prática é firmemente condenada.

Diferentemente dos trechos apresentados anteriormente, o próximo recorte critica tanto a teoria como a prática da inclusão.

(4) Ela pergunta: "Por que a inclusão na Alemanha ainda é tão controversa?” Ainda? Cada vez mais! Cada vez mais se torna evidente o caráter utópico e irreal de uma ideia de cura, que não possui um conceito positivo da desigualdade. (FAZ.NET, 21/07/2014; tradução própria).

(Original em alemão: Sie fragt: „Warum ist die Inklusion in Deutschland noch immer so umstritten?" Noch immer? Immer mehr! Immer mehr zeigt sich der utopische, weltfremde Charakter einer Heilsidee, die über keinen positiven Begriff von Ungleichheit verfügt.)

Verifica-se que, além da atribuição utópica e irreal, a classificação da inclusão como ideia de cura, por um lado, sublinha novamente o caráter teórico e imaginário da conceitualização. Por outro lado, pelo uso irônico dessa expressão, atesta-se uma caraterização negativa da inclusão.

Nos exemplos seguintes, coloca-se a conceitualização da inclusão como ilusão no centro da análise. Em alemão, a palavra correspondente, Illusion, denomina um autoengano eufemístico sobre fatos que, na realidade, são menos positivos. Portanto, esse autoengano surge de um pensamento influenciado por um desejo (DUDENREDAKTION, 2016). Sendo essa percepção da inclusão bastante popular no discurso midiático, verifica-se a sua aparência até no título de um artigo:
(5) A ilusão com a inclusão (FAZ. NET, 24/05/2014; tradução própria).

(Original em alemão: Die Illusion mit der Inklusion).

Por consequência, constata-se que a inclusão no discurso alemão está classificada como um estado que somente parece desejável, porque seus defensores estão sujeitos a um autoengano sobre a realidade. Todavia, não sempre esse autoengano se refere à inclusão em si:

(6) Menos dotados devem aprender dos dotados. Sob essas condições é uma ilusão! (BILD.DE, 20/02/2015; tradução própria).

(Original em alemão: Weniger Begabte sollen von Begabten lernen. Das ist unter diesen Voraussetzungen eine Illusion!)

Para um entendimento melhor do exemplo seguinte, convém mencionar que o artigo do qual foi recortado tematiza o mau equipamento e a falta de capacitação dos professores para a educação inclusiva. Em face de que a conceitualização como ilusão nesse fragmento discursivo se restringe à inclusão sob ditas condições, percebe-se que o aspecto irreal se refere somente a esses casos. Seguindo o pensamento de tal artigo, verifica-se que, no caso contrário, se as escolas e os professores fossem preparados, a inclusão seria realizável.

Outra observação da análise é a tematização da desilusão quanto à inclusão:

(7) As primeiras ilusões estouraram. Também as dos alunos. Se ele pudesse decidir novamente, ele não voltaria à classe inclusiva, diz Kornelius [...].(ZEIT ONLINE, 30/07/2015; tradução própria).

(Original em alemão: Die ersten Illusionen sind geplatzt. Auch bei den Schülern. Könnte er noch mal entscheiden, er würde nicht wieder in die Inklusionsklasse gehen, sagt Kornelius [].)

Mesmo que o artigo aborde as ilusões estouradas ao realizar a inclusão, verifica-se um fortalecimento 
do conceito da inclusão como ilusão, já que essa circunstância pode ser entendida como uma prova de que a educação inclusiva é um projeto irreal.

Outro conceito da inclusão que se encaixa semanticamente nas conceitualizações aqui já tratadas, é a inclusão como visão. A palavra visão, Vision em alemão, denomina uma imagem, principalmente sobre o futuro, criada na imaginação de alguém (DUDENREDAKTION, 2016). Novamente, nota-se o caráter imaginário do conceito, embora, diferentemente dos outros conceitos aqui descritos, não se indique se se trata de uma imagem real(izável) ou irreal(izável). Destarte, no caso da palavra visão, é o contexto que esclarece esse detalhe do conceito:

(8) Como uma visão é agradável: todas as escolas ensinam individualmente. Cada professor tem o tempo e o treinamento para atender todas as crianças. [...] Mas, em primeiro lugar, isto não corresponde à realidade, e, em segundo lugar, consequentemente, teríamos de admitir que Gymnasien e Realschulen seriam supérfluas. (ZEIT ONLINE, 19/05/2014; tradução própria).

(Original em alemão: Als Vision ist das zwar schön: Alle Schulen unterrichten individuell. Jeder Lehrer hat die Zeit und die Ausbildung, jedem Kind gerecht zu werden. []

Aber erstens entspricht das nicht der Realität und zweitens müsste man dann konsequenterweise zugeben, dass Gymnasien und Realschulen überflüssig wären).

Nesse trecho, os leitores deparam novamente com o esquema já observado nas conceitualizações anteriores: uma avaliação positiva da teoria da inclusão que não corresponde à realidade. Focando a segunda parte da argumentação do exemplo, nota-se que ela discorre sobre a incompatibilidade do sistema educacional alemão com a educação inclusiva. Perante isso, o artigo afirma que a inclusão revogaria a realidade educacional atual. Portanto, pode-se deduzir que a conceitualização da inclusão como algo irreal ocorre - entre outras causas - pelo motivo de que (uma parte d)a sociedade alemã não está dispos- ta a esse passo. Pode-se deduzir, também, que esse é um dos motivos da avaliação negativa da inclusão no discurso midiático:

(9) Uma escola para todos, essa é a visão que visa a permitir a arma mágica pedagógica da inclusão. Em algumas salas de aula, ela já se tornou um pesadelo. (ZEIT ONLINE, 09/06/2011; tradução própria).

(Original em alemão: Eine Schule für alle, das ist die Vision, die die pädagogische Wunderwaffe Inklusion ermöglichen soll. In manchen Klassenzimmern ist sie schon jetzt zum Albtraum geworden.)

Frisa-se o caráter representativo da escola para todos como símbolo da educação inclusiva no discurso midiático, lembrando que esta, por exemplo, no Trecho (1), foi denominada a precursora da inclusão social total. Nesse fragmento (10), ao contrário, ela é o meio para poder efetivar a inclusão. Porém, conforme o artigo, esse meio não possui um caráter real, visto que é considerado uma visão e uma arma mágica. Esta última expressão contém, adicionalmente ao aspecto irreal, uma conotação negativa, sendo associada com violência e guerra. De maneira semelhante, a palavra pesadelo contribui para a conceitualização negativa da inclusão, mantendo ao mesmo tempo seu atributo de irrealidade.

No trecho seguinte, verifica-se um posicionamento que se opõe à tendência do discurso:

(10) A inclusão significa o reconhecimento e a valorização de pessoas com deficiência.

Essa é uma grande reivindicação, uma meta muito ambiciosa. É uma visão real - que a constituição alemã formula há 20 anos, desde 1994, da seguinte maneira: "Ninguém pode ser discriminado por causa de sua deficiência." (SZ.DE, 16/06/2016; tradução própria).

(Original em alemão: Inklusion heißt Anerkennung und Wertschätzung für Menschen mit Behinderungen. Das ist ein gewaltiger Anspruch, das ist ein hochgestecktes Ziel. Es ist eine Realvision - die das Grundgesetz seit 20 Jahren, seit 1994, so formuliert: "Niemand darf wegen seiner Behinderung benachteiligt werden.")

Levando em conta que a definição da palavra visão em alemão se refere a uma imagem principalmente 
sobre o futuro criada na imaginação de alguém, nota-se que essa descrição deixa aberto o aspecto da viabilidade. Dessa vez, no exemplo (11), percebe-se que tanto a atribuição real como o contexto retiram o aspecto imaginário e irreal do conceito da inclusão. Mencionando a constituição, o trecho ainda desloca a visão do futuro ao presente. Diante disso, pode-se constatar a tentativa no discurso de desconstruir a conceitualização do fator irreal da inclusão, retomando e ressignificando um conceito utilizado pelos adversários da inclusão.

\section{CONSIDERACÕ̃ES FINAIS}

Ao considerar os resultados da análise, verifica-se no discurso midiático alemão a construção de uma imagem da inclusão que a deixa parecer um ideal irrealizável. Essa construção efetua-se por meio de uma rede de conceitos, resultado de processos da integração conceitual, combinando a educação inclusiva com conceitos do irreal e do imaginário, principalmente a utopia, a ilusão e a visão. Em consequência, a inclusão passa por um processo de significação. Constata-se que, em alguns casos, constrói-se um contraste entre a teoria e a prática da inclusão, caraterizando a teoria de uma maneira positiva e como um futuro desejável, enquanto a prática é definida como irreal.

Ainda assim, quando se contempla a inclusão praticada, salienta-se a inviabilidade da inclusão por meio de experiências negativas, determinando-as como pesadelos ou alertando para seus efeitos colaterais. Em outros casos, critica-se tanto a teoria como a prática. Porém, vale ressaltar que em dois exemplos se observam tendências que não se encaixam completamente nessa conceitualização.

Em um recorte, o aspecto irreal se refere somente à inclusão escolar sob condições insuficientes nas escolas e na formação dos professores. O outro caso citado contradiz o construto da inclusão como um projeto irreal e um assunto futuro, remetendo, por um lado, para um parágrafo da constituição alemã que proíbe a discriminação de pessoas com deficiência desde 1994 e, por outro lado, retomando e ressignificando o conceito discursivo da inclusão como visão. Todavia, salienta-se que esses dois exemplos representam exceções.

Ao levar em conta a teoria do discurso da ACD, convém refletir sobre as consequências e os motivos sociais dessa rede de conceitualizações. Discorrendo sobre a influência da sociedade sobre o discurso, nota-se que a percepção da inclusão como algo irreal e irrealizável demonstra que a sociedade alemã - ou pelo menos uma parte dela - não aceita uma mudança social quanto ao sistema educacional. Tendo em vista a peculiaridade do sistema escolar, nota-se que a mudança para um sistema inclusivo, no caso alemão, significa uma reestruturação total. Salienta-se que, segundo princípios democráticos, tais mudanças precisam ser legitimadas pelo consentimento da sociedade, o que, por sua vez, não se pôde verificar pela conceitualização dominante no discurso.

Em consideração da influência do discurso sobre a sociedade, enfatiza-se a (re)produção de certo significado da inclusão, sendo esta definida como irreal. Em vista de que os meios de comunicação de massa atingem uma grande parte da sociedade, eles desempenham um papel importante na construção de verdades sociais. Destarte, pode-se constatar que o discurso midiático contribui para a recusa social da inclusão praticada em escolas, (re)construindo e distribuindo um conceito da inclusão que nega a sua viabilidade.

Consequências possíveis são, em primeiro lugar, um atraso na realização da Convenção da ONU e, portanto, no estabelecimento dos direitos das pessoas com necessidades educacionais especiais; em segundo lugar, essa conceitualização discursiva também pode ter efeitos sobre a (auto)percepção das pessoas com necessidades educacionais especiais, visto que estas são - indiretamente - definidas como tão diferentes que a educação inclusiva é impossível. 


\section{REFERÊNCIAS}

\begin{abstract}
ALEMANHA. Inklusive Bildung von Kindern und Jugendlichen mit Behinderungen in Schulen. Beschluss der Kultusministerkonferenz vom 20.10.2011. Disponível em: <https://www.kmk.org/ dokumentation-und-statistik/beschluesse-und-veroeffentlichungen/bildung-schule/allgemeine-bildung. html\#c1315>. Acesso em: 2 maio 2016.
\end{abstract}

AUTORENGRUPPE BILDUNGSBERICHTERSTATTUNG. Bildung in Deutschland 2014. Ein indikatorengestützter Bericht mit einer Analyse zur Bildung von Menschen mit Behinderungen. Bielefeld: Bertelsmann, 2014.

BERLIM. Schulgesetz für das Land Berlin (Schulgesetz - SchulG). Vom 26. Januar 2004. Gesamtausgabe in der Gültigkeit vom 06.04.2016 bis 14.08.2016. Berlim, 2004. Disponível em: <http://gesetze.berlin.de/jport $\mathrm{al} /$ ?quelle=jlink\&query=SchulG+BE\&psml=bsbeprod. psml\&max=true\&aiz=true>. Acesso em: 22 abr. 2016.

BILD.DE. Berlin: Springer. Disponível em: http:// www.bild.de. Acesso em: 06 maio 2016.

\section{BRASIL. Convenção sobre os Direitos das Pessoas com Deficiência. 4.ed. Brasília, 2012.}

DUDENREDAKTION. Duden online. Berlin: Bibliographisches Institut, 2016. Disponivel em:< http://www. duden.de>. Acesso em: 26 abr. 2016.

FAIRCLOUGH, Norman. Globaler Kapitalismus und kritisches Diskursbewusstsein. In: KELLER, Reiner et al. (Hg.). Handbuch sozialwissenschaftliche Diskursanalyse. Band 1: Theorien und Methoden. Wiesbaden: VS Verlag für Sozialwissenschaften, 2006, p. 339-55.

FAIRCLOUGH, Norman; WODAK, Ruth. Critical Discourse Analysis. In: VAN DIJK, Teun (org.). Discourse as Social Interaction. Discourse studies: A multidisciplinary introduction. V.2. London: Sage, 1997. p.258-284.

FAUCONNIER, Gilles; TURNER, Mark. The way we think. Conceptual Blendings and the mind's hidden complexities. New York: Basic Books, 2002.

FAUCONNIER, Gilles; TURNER, Mark. Rethinking Metaphor. In: GIBBS, Ray (Ed.). Cambridge Handbook of Metaphor and Thought. Cambridge: University Press, 2008.

FAZ.NET. Frankfurt: Frankfurter Allgemeine Zeitung. Disponível em: <http://www.faz.net/>. Acesso em: 6 maio 2016.

FERRARI, Lilian. Introdução à linguística cognitiva. São Paulo: Contexto, 2014.

FRANK, Roslyn M. et al. (Ed.). Body, language and mind. V.2: Sociocultural situatedness. Berlin; New York: de Gruyter, 2008.

HART, Christopher. Event-construal in press reports of violence in political protests: a cognitive linguistic approach to CDA. Journal of Language and Politics, v.12, n.3, 2013. p.400-423.

KRÖG, Walter. Einleitung. In: MENSCH IM MITTELPUNKT (MIM); VEREIN TAFIE AUSSERFERN (Hg.).

Herausforderung Unterstützung: Perspektiven auf dem Weg zur Inklusion. Lechaschau: MiM/TAFIE, 2005. p.3-6.

LAKOFF, George; JOHNSON, Mark. Metaphors we live by. Chicago / London: University of Chicago Press, 1980.

LAKOFF, George; JOHNSON, Mark. Philosophy in the flesh. The embodied mind and its challenge to Western thought. New York: Basic Books, 1999. 
MISSLING, Sven; ÜCKERT, Oliver. Inklusive Bildung: Schulgesetze auf dem Prüfstand. Berlin: Deutsches Institut für Menschenrechte, 2014. Disponível em: <http://www.institut-fuer-menschenrechte.de/ monitoring-stelle-un-brk/publikationen/>. Acesso em: 3 jan. 2016.

RATZKI, Anne. Gesamtschule - Gemeinschaftsschule: Aktuelle bildungspolitische Herausforderungen. In: AUFTAKTVERANSTALTUNG ZUR KAMPAGNE NEUE GESAMTSCHULEN FÜR KÖLN, 2011. Disponível em: <http://www.hf.unikoeln.

de/data/gbd/File/Anne\%20Ratzki_2011_Gesamtschule_Gemeinschaftsschule.pdf>. Acesso em: 22 abr. 2016.

SCHUMANN, Brigitte. “Ich schäme mich ja so!” Die Sonderschule für Lernbehinderte als "Schonraumfalle”. Bad Heilbrunn: Klinkhardt, 2007.

SZ.DE. München: Süddeutscher Verlag. Disponível em: <http://www.sueddeutsche.de>. Acesso em: 6 maio 2016.

\section{UNESCO. International Standard Classification of} Education: ISCED 2011. Montreal; Quebec: UIS, 2012.
VAN DIJK, Teun A. Political discourse and political cognition. In: CHILTON, Paul A.; SCHÄFFNER, Christina (Ed.). Politics as text and talk. Analytical approaches to political discourse. Amsterdam: Benjamins, 2002. p.204-236.

VAN DIJK, Teun A. The discourse-knowledge interface. In: WEISS, Gilbert; WODAK, Ruth (Ed.). Critical

Discourse Analysis. Theory and interdisciplinarity. Houndsmills: Palgrave Macmillan, 2003. p.85-109.

VIOTTI, Maria Luiza Ribeiro. A educação básica e o ensino médio na Alemanha. In: BRASIL. Coleção

Mundo Afora no 11: Educação Básica e Ensino. Brasília, 2014, p. 22-33. Disponível em: <http:// dc.itamaraty.gov.br/publicacoes/mundo-afora-no-11-educacao-basica-e-ensino-medio>. Acesso em: 3 jan. 2016.

WODAK, RUTH. Critical Linguistics and Critical Discourse Analysis. In: VERSCHUEREN, Jef; ÖSTMAN, Jan-Ola; BLOMMAERT, Jan (eds.): Handbook of Pragmatics: Manual. Amsterdam; Philadelphia: Benjamins, 1995. p.204-210.

ZEIT ONLINE. Hamburg: Zeitverlag. Disponível em: <http://www.zeit.de>. Acesso em: 6 maio 2016. 